\title{
Interactions between holocellulose and lignin during hydrolysis of sawdust in subcritical water
}

Wei Yanga ${ }^{\mathrm{a}}$, Munan Fanga ${ }^{\mathrm{a}}$, Hao Xu ${ }^{\mathrm{b}}$, Hui Wang ${ }^{\mathrm{a}}$, Shengji Wu ${ }^{\mathrm{a}, *}$, Jie Zhou ${ }^{\mathrm{a}}$, Shouxin Zhu ${ }^{\mathrm{b}}$

${ }^{a}$ College of Materials and Environmental Engineering, Hangzhou Dianzi University, Xiasha University

Park, Hangzhou, Zhejiang 310018, China

${ }^{b}$ College of Engineering, Huzhou University, No. 759, East 2nd Road, Huzhou, 313000 China

*Corresponding author: Tel: +86 (0) 571-8691-5125; E-mail: wushengji26@hdu.edu.cn 
Isolation of holocellulose and lignin from sawdust. Holocellulose was isolated from sawdust by an acid-chlorite delignification method. $100 \mathrm{mg}$ of sawdust combined with $4 \mathrm{~mL}$ of deionized water was charged into an eggplant type flask, and reacted with $200 \mathrm{mg}$ of $80 \%$ sodium chlorite and $0.8 \mathrm{~mL}$ of acetic acid at $90{ }^{\circ} \mathrm{C}$ for $1 \mathrm{~h}$. The suspension was then filtered by a pre-weighted G-4 sand-core funnel, washed by $50 \mathrm{~mL}$ of deionized water for 3 times and dried at $105{ }^{\circ} \mathrm{C}$ for $24 \mathrm{~h}$. The dried solid was holocellulose. Lignin was purified by a $72 \%$ sulfuric acid method. 100 $\mathrm{mg}$ of sawdust was treated by $1.5 \mathrm{~mL}$ of $72 \%$ sulfuric acid at ambient temperature for $2 \mathrm{~h}$ to hydrolyze and solubilize carbohydrates. Then, $56 \mathrm{~mL}$ of deionized water was added to reduce the sulfuric acid concentration to $3 \%$ and the suspension was further boiled for $4 \mathrm{~h}$. After that, the boiled suspension was filtered by a pre-weighted G-4 sand-core funnel, and washed by hot water until reaching a neutral $\mathrm{pH}$. 
Table S1. Coats-Redfern method kinetic expressions.

\begin{tabular}{cc} 
Model & $g(\alpha)$ \\
\hline \multicolumn{2}{c}{ Diffusion Models } \\
One-way transport (D1) & $\alpha^{2}$ \\
Two-way transport (D2) & {$[(1-\alpha) \ln (1-\alpha)]+\alpha$} \\
Three-way transport (D3) & {$\left[\left(1-(1-\alpha)^{1 / 3}\right)\right]^{2}$} \\
Ginstling-Brounshtein (D4) & $\left(1-(2 \alpha / 3)-(1-\alpha)^{1 / 3}\right)$ \\
Geometrical Contraction Models \\
Contracting model (R2) & $1-(1-\alpha)^{1 / 2}$ \\
Contracting volume (R3) & $1-(1-\alpha)^{1 / 3}$ \\
Reaction Order Models \\
First-order (F1) & $-\ln (1-\alpha)$ \\
Second-order (F2) & $(1-\alpha)^{-1}-1$ \\
Third-order (F3) & {$\left[(1-\alpha)^{-2}-1\right] / 2$} \\
\hline
\end{tabular}


Table S2. Ethyl acetate soluble products in the hydrolysates derived from holocellulose and lignin after subcritical water treatment at $273^{\circ} \mathrm{C}$.

\begin{tabular}{|c|c|c|c|c|c|}
\hline \multirow{2}{*}{ Compounds } & \multicolumn{2}{|c|}{ Area yield (\%) } & \multirow{2}{*}{ Compounds } & \multicolumn{2}{|c|}{ Area yield (\%) } \\
\hline & Holocellulose & Lignin & & Holocellulose & Lignin \\
\hline Isopropyl acetate & 0.08 & 0.15 & Orcinol & 0.08 & - \\
\hline $\begin{array}{l}\text { Propanoic acid, } \\
\text { ethyl ester }\end{array}$ & 0.11 & 0.15 & $\begin{array}{l}\text { 2-Methoxy-4- } \\
\text { vinyphenol }\end{array}$ & 0.01 & 0.01 \\
\hline Furan, 3-methyl & 0.05 & - & $\begin{array}{l}\text { Phenol, 2-methoxy- } \\
\text { 5-(1-propenyl)- }\end{array}$ & - & - \\
\hline Furfural & 12.47 & 0.46 & Vanillin & 0.02 & 0.13 \\
\hline $\begin{array}{l}\text { Ethanone, 1-(2- } \\
\text { furanyl)- }\end{array}$ & 0.05 & - & $\begin{array}{l}\text { Phenol, 2-methoxy- } \\
\text { 4-(1-propenyl)- }\end{array}$ & - & - \\
\hline $\begin{array}{l}\text { Furancarboxaldehy } \\
\text { de, 5-methyl- }\end{array}$ & 0.29 & 0.04 & $\begin{array}{c}\text { Phenol, 2-methoxy- } \\
\text { 4-propyl- }\end{array}$ & 0.01 & 0.09 \\
\hline $\begin{array}{l}\text { 2-Acetyl-5- } \\
\text { methylfuran }\end{array}$ & 0.01 & - & Coniferyl aldehyde & - & 0.02 \\
\hline $\begin{array}{c}\text { 5-Acetoxymethyl-2- } \\
\text { furaldehyde }\end{array}$ & 0.11 & - & $\begin{array}{l}\text { 1-(4-hydroxy-3- } \\
\text { methoxyphenyl)buta } \\
\text { n-1-one }\end{array}$ & 0.1 & 0.1 \\
\hline $\begin{array}{c}\text { Furyl } \\
\text { hydroxymethyl }\end{array}$ & 0.4 & - & $\begin{array}{l}\text { 2-(1-Cyclohexenyl) } \\
\text { cyclohexanone }\end{array}$ & 0.02 & 0.01 \\
\hline $\begin{array}{c}5- \\
\text { Hydroxymethylfurf } \\
\text { ural }\end{array}$ & 11.33 & 0.05 & $\begin{array}{l}\text { Hexanoic acid, 2- } \\
\text { ethyl-, oxybis(2,1- } \\
\text { ethanediyloxy-2,1- } \\
\text { ethanediyl) ester }\end{array}$ & 0.11 & - \\
\hline Levoglucosenone & 0.1 & - & $\begin{array}{l}\text { Benzenepropanol, 4- } \\
\text { hydroxy-3-methoxy- }\end{array}$ & 0.02 & - \\
\hline Phenol, 2-methoxy- & 0.12 & 0.05 & $\begin{array}{l}\text { Benzene, 3-ethyl- } \\
\text { 1,2,4,5-tetramethyl }\end{array}$ & - & 0.01 \\
\hline
\end{tabular}






Figure S1. Flow scheme of subcritical water hydrolysis of sawdust, holocellulose, lignin and holocellulose-lignin mixture. 


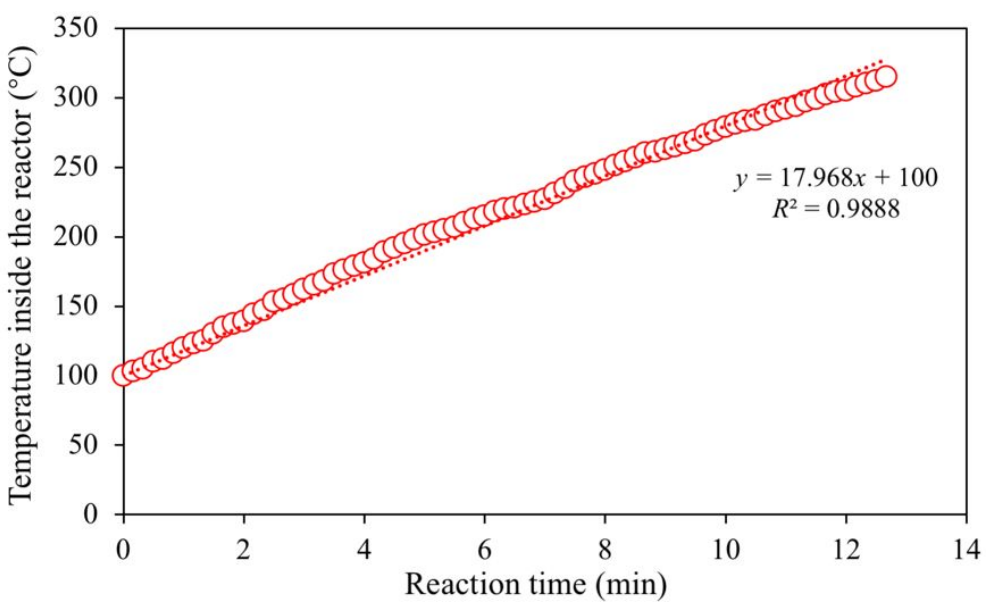

Figure S2. Temperature inside the reactor at various residence times 



Figure S3. Relationship between experimental data of hydrolysis (a and b) sawdust, (c and d) holocellulose and (e and f) holocellulose-lignin mixture and linear fitted lines based on kinetic models. 


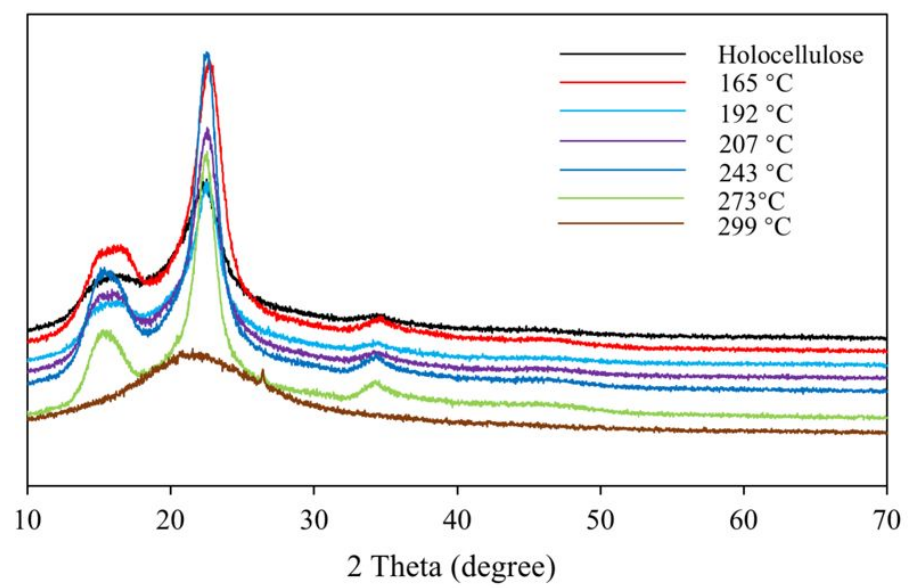

Figure S4. XRD measurement of holocellulose and its derived residues. 

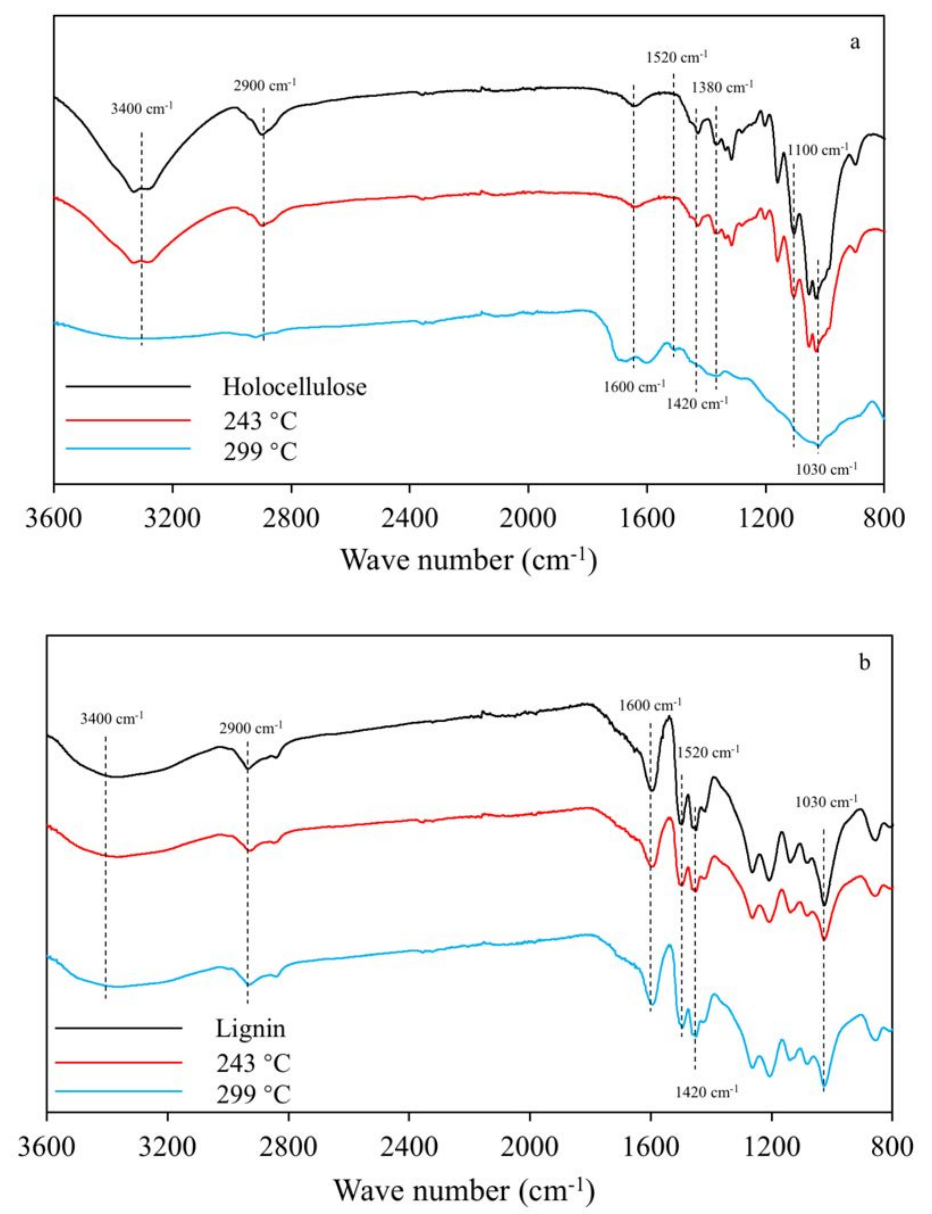

Figure S5. FTIR measurement of (a) holocellulose and its derived residues, and (b) lignin and its derived residues. 

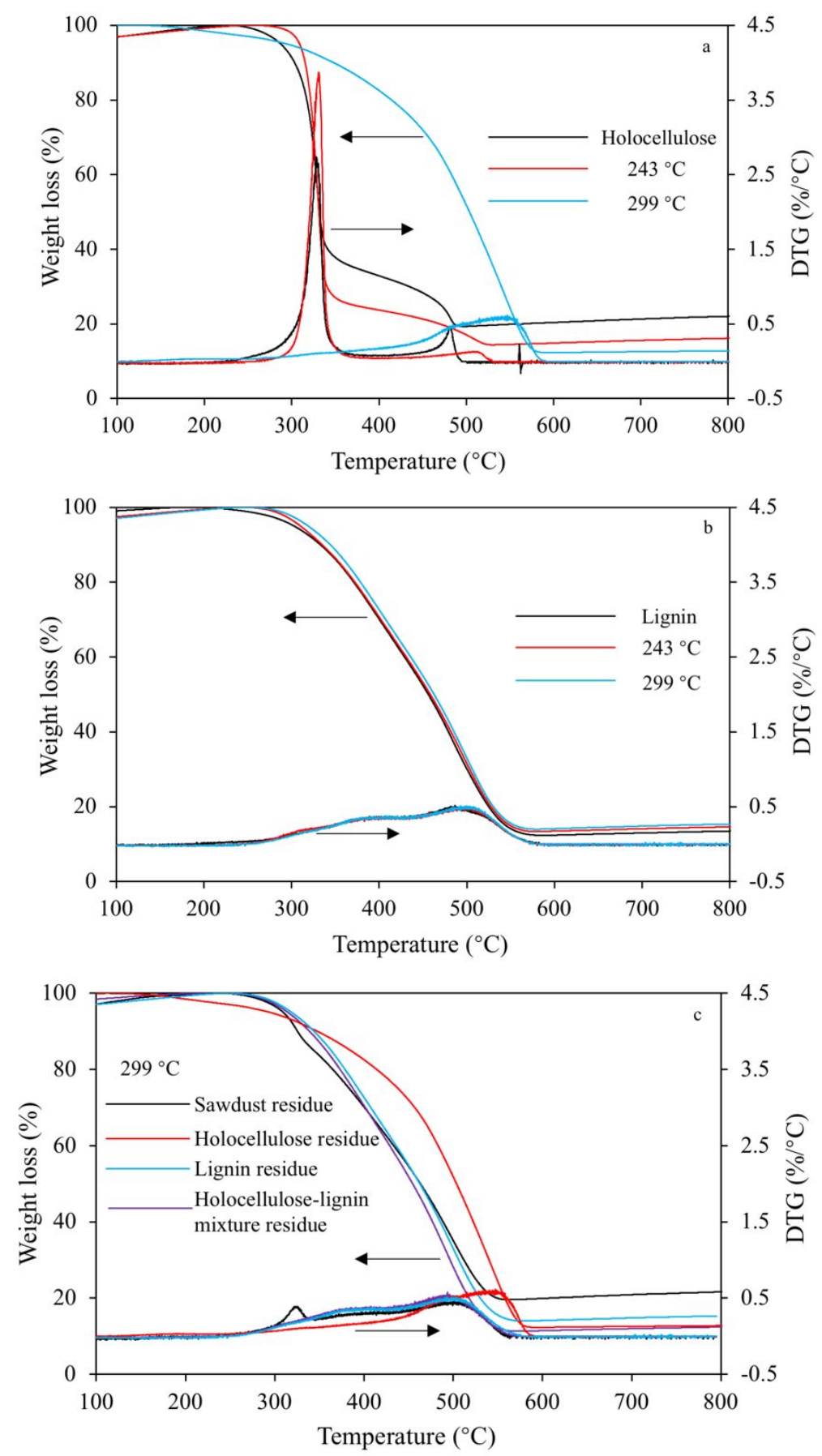

Figure S6. TGA measurement of (a) holocellulose and its derived residues, (b) lignin and its derived residues and (c) sawdust, holocellulsoe, lignin and holocellulose-lignin mixture residues obtained at $299^{\circ} \mathrm{C}$. 

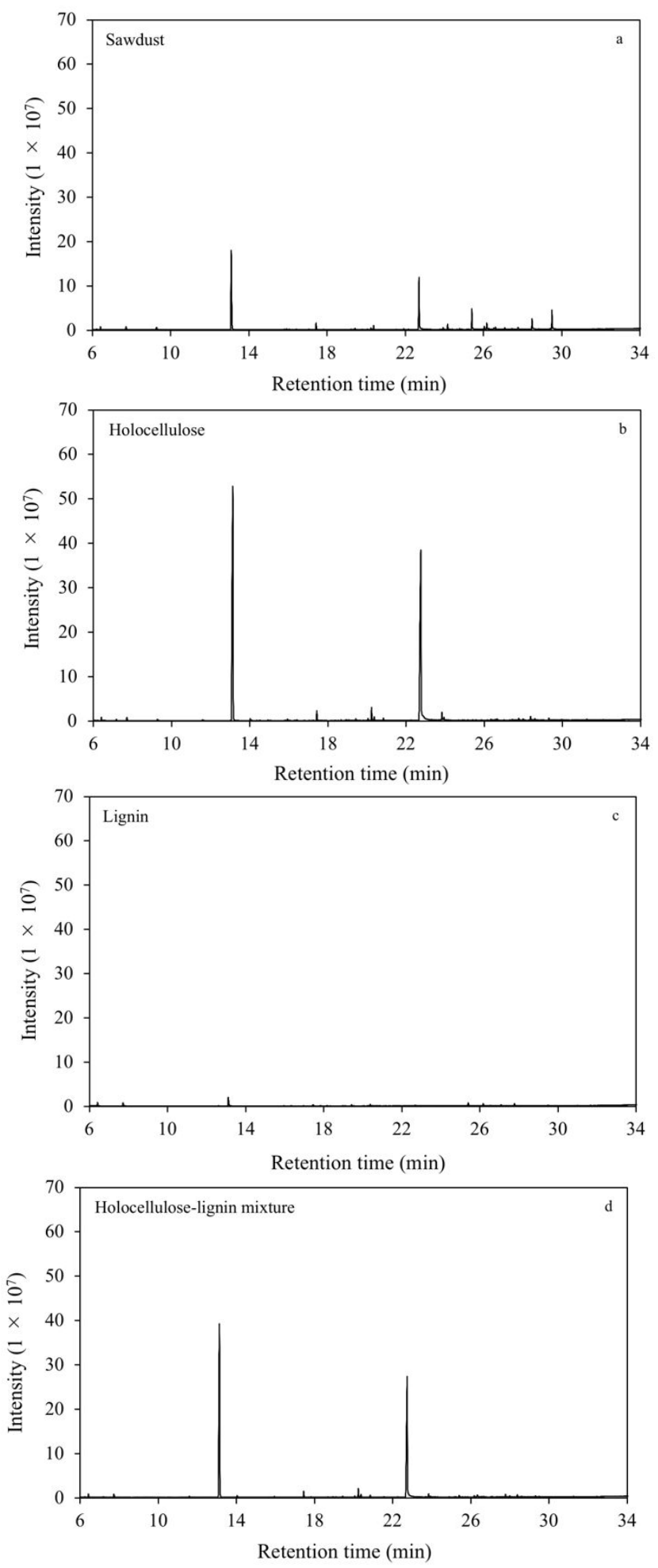

Figure S7. GC/MS chromatographs of ethyl acetate soluble products derived from (a) sawdust, (b) holocellulose, (c) lignin and (d) holocellulose-lignin mixture treated at $273{ }^{\circ} \mathrm{C}$. 


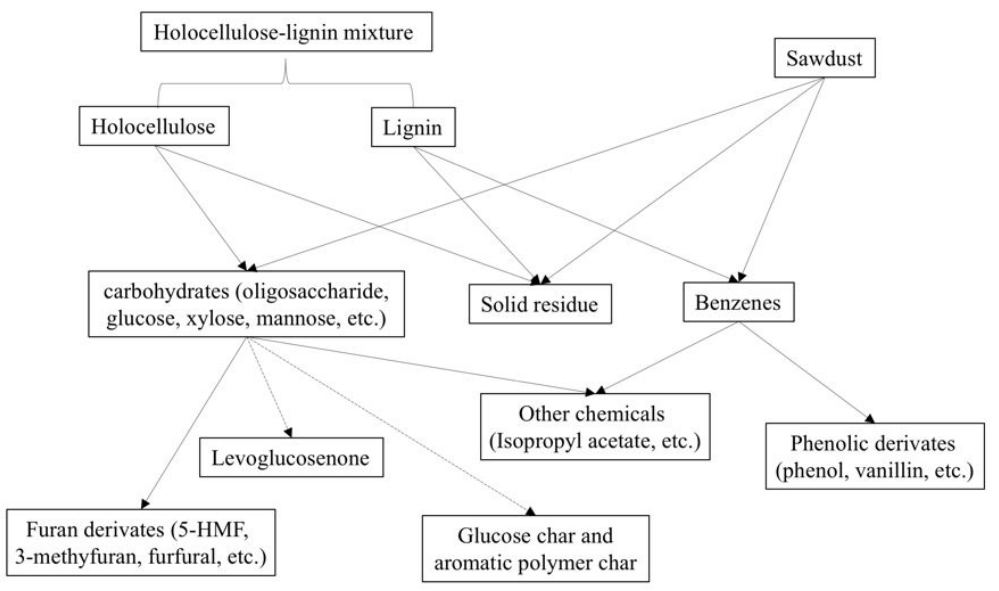

Figure S8. Simplified reaction paths for sawdust and holocellulose-lignin mixture in subcritical water. (The dotted arrow was the suppressed reaction path.) 